\title{
Signal-to-noise ratio of single-pixel cameras based on photodiodes
}

\author{
Y. Jauregui-Sánchez ${ }^{1,}{ }^{*}$, P. Clemente ${ }^{2}$, P. Latorre-Carmona ${ }^{3}$, E. Tajahuerce ${ }^{1}$, and J. Lancis ${ }^{1}$ \\ ${ }^{1}$ GROC.UJI, Institute of New Imaging Technologies (INIT), Universitat Jaume I, 12071, Castelló, \\ Spain \\ ${ }^{2}$ Servei Central d'Instrumentació Científica (SCIC), Universitat Jaume I, 12071, Castelló, Spain \\ ${ }^{3}$ eVIS, Institute of New Imaging Technologies (INIT), Universitat Jaume I, 12071 Castelló, Spain \\ ${ }^{*}$ Corresponding author: jauregui@uji.es
}

\begin{abstract}
Single-pixel cameras have been successfully used in different imaging applications in the last years. One of the key elements affecting the quality of these cameras is the photodetector. Here we develop a numerical model of a single-pixel camera, which takes into account not only the characteristics of the incident light but also the physical properties of the detector. In particular, our model considers the photocurrent, the dark current, the photocurrent shot noise, the dark current shot noise, and the Johnson-Nyquist (thermal) noise of the photodiode used as light detector. The model establishes a clear relationship between the electric signal and the quality of the final image. This allows us to perform a systematic study of the quality of the image obtained with single-pixel cameras in different contexts. In particular, we study the signal-to-noise ratio (SNR) as a function of the optical power of the incident light, the wavelength, and the photodiode temperature. The results of the model are compared with those obtained experimentally with a single-pixel camera.
\end{abstract}

Keywords: Imaging systems; Computational imaging; Spatial light modulators; Photodetectors; Noise in imaging systems.

https://doi.org/10.1364/A0.57.000B67

\section{Introduction}

Computational imaging with a single-pixel camera, or single-pixel imaging (SPI), is a remarkable alternative to conventional imaging [1]. The technique is based on sampling the object with a sequence of microstructured light patterns codified onto a programmable spatial light modulator (SLM) while light intensity is measured with a singlepixel detector. The image is computationally retrieved from the photocurrent fluctuations provided by the detector.

The simplicity of the detection stage in SPI is one of the main advantages of the technique. It can be exploited to use very sensitive light sensors in low light level applications [2]. It is also useful to measure the spatial distribution of different parameters such as the spectral content $[2,3]$ or the polarization state [4] of the incident light. Furthermore, single-pixel techniques are very well suited for the application of compressive sampling methods, commonly known as compressive sensing, which noticeably reduce the acquisition time [5].

During the last decade SPI has been successfully applied in many different imaging scenarios. Among them we can mention infrared imaging [6,7], terahertz imaging [8], 3D computational imaging $[9,10]$, imaging through scattering media [11-13], 3D and photon counting light detection and ranging (LIDAR) imaging systems [14-16], stereoscopic imaging [17], microscopy [18,19], ultrasonic imaging [20], and ophthalmoscope imaging [21]. Moreover, there are also several studies related to the SNR of single-pixel 
cameras [22-24]. However they do not do a rigorous study of the performance of single-pixel cameras based on the physical parameters of the detector.

One of the key elements in single-pixel imaging is, obviously, the photodetector. Among the different possible photodetectors, photodiodes are the most common sensors in general single-pixel imaging applications. A photodiode is a semiconductor device that converts the optical signal in a current one by electronic processes [25]. Therefore, the electric signal quality can be analyzed by determining different currents and noise sources, for instance, the photocurrent, the dark current, the photocurrent shot noise, the dark current shot noise, and the Johnson-Nyquist (thermal) noise.

In this paper we develop a numerical model of a single-pixel camera, which takes into account not only the characteristics of the incident light but also the physical features of the photodiode. The model examines the relationship between the properties of the optical signal, the electric signal, and the quality of the final image. In particular, we study the SNR as a function of the optical power and the wavelength of the incident light, and the photodiode temperature. We restrict our study to silicon ( $\mathrm{Si}$ ) and indiumgallium-arsenide (InGaAs) photodiodes because those are commonly used in the visible and the infrared spectral regions.

In the following sections, firstly, we develop a mathematical review of the electrical behavior of photodiodes, in particular those based on Si and InGaAs materials. Second, we describe the numerical model of the single-pixel camera. Next, we apply this model to study the SNR of a single-pixel camera in different contexts. We compare some of these results with those obtained experimentally in the laboratory. Finally, we emphasize the main conclusions.

\section{The photodiode signal in a single-pixel camera}

In single-pixel imaging, an image is reconstructed from the signal generated by the light transmitted or reflected by the object when it is sequentially sampled with a set of microstructured patterns. This can be done by codifying the input light beam with a spatial light modulator (SLM) and projecting the light patterns onto the object, or by employing a uniform light beam and projecting an image of the object onto the SLM. Fig. 1 shows the first approach by a trans-illumination configuration. In this case, the SLM, a digital micromirror device (DMD), is illuminated with the light produced by a white-light source, conveniently expanded, homogenized, and filtered. Afterwards, the microstructured light patterns codified on the DMD display are projected onto the object using a 4-f optical system formed by two lenses. A circular diaphragm at the focal plane filters unwanted diffracted orders. Then, the light transmitted by the object is collected and focused by a lens onto the photodetector. In order to numerically recover the image, the current signal is digitized by a data acquisition system (DAQ) and processed in a computer.

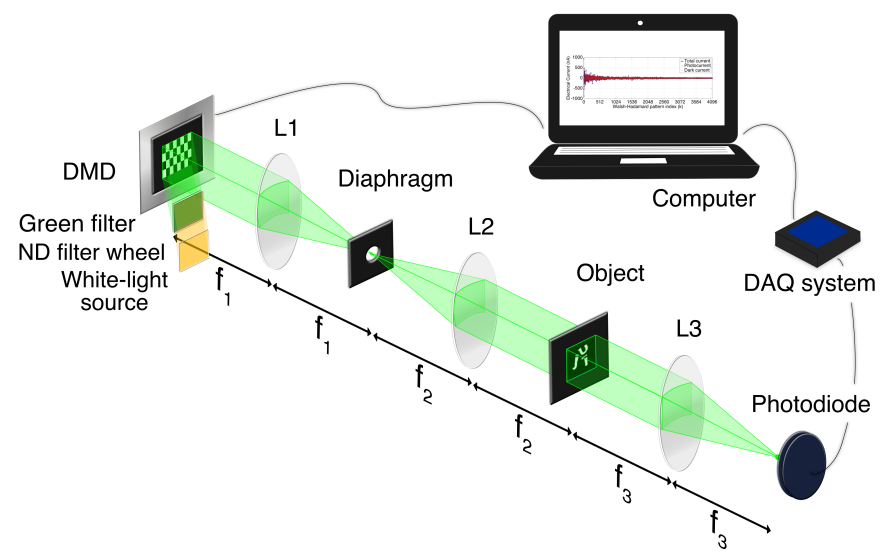

Figure 1: Schematic diagram of a single-pixel camera setup.

The correlation level between each light pattern and the object directly determines the photodiode electrical current. Therefore, the quality of the image depends on the characteristics of the photocurrent signal. In order to show this relation, we study the photodiode signal and its noise sources. The photodiode signal is composed by two terms, the photocurrent $\left(I_{P}\right)$ and the dark current $\left(I_{D}\right)$. The first one is due to the photoelectric effect on the photodiode surface and it is given by [26],

$$
I_{P}=R_{\lambda} \cdot P
$$

where $\mathrm{P}$ is the optical power level of the light source and $R_{\lambda}$ is the photodiode responsivity $\left[\frac{A}{W}\right]$. The second current is always present in the photodiode, even without illumination, and it is originated by the thermal generation of electron-hole pairs in the Si p-n and InGaAs p-i-n photodiode layers. Four sources contribute to the dark current: the generation-recombination current in the depletion region, the diffusion current from the undepleted regions, the tunneling current, and the surface leakage current [27-29]. Nevertheless, the current-voltage of a p-n diode can be ideally described by the Shockley equation, which is given by $[25]$

$$
I_{D}=I_{s}(T)\left[e^{\left(\frac{q V_{A}}{k_{B} T}\right)}-1\right]
$$

where $q=1.602 \times 10^{-} 19 C$ is the electron charge, $V_{A}$ is the 
bias voltage, $k_{B}=1.381 \times 10^{-23} \frac{\mathrm{J}}{\mathrm{K}}$ is the Boltzmann's constant, and $T$ is the absolute temperature. Then, under the reverse-bias condition, the saturation current (as a function of the temperature $T$ ) can be written as

$$
I_{s}(T)=C_{n} A_{P} T^{3} e^{\left(-\frac{E_{g}(T)}{k_{B} T}\right)}+C_{n} A_{P} T^{\frac{3}{2}} e^{\left(-\frac{E_{g}(T)}{2 k_{B} T}\right)} .
$$

Assuming the temperature is lower or close to room temperature, the first term in Eq.(3) can be considered negligible [25]. Taking this into account and substituting this expression in Eq.(2), the dark current is given by

$$
I_{D}=C_{n} A_{P} T^{\frac{3}{2}} e^{\left(-\frac{E_{g}(T)}{2 k_{B} T}\right)}\left[e^{\left(\frac{q V_{A}}{k_{B} T}\right)}-1\right]
$$

where $C_{n}$ is a constant factor $\left[\frac{A}{\mathrm{~cm}^{2}}\right]$ and $A_{P}$ is the photodiode area $\left[\mathrm{cm}^{2}\right] . E_{g}(T)$ is the band gap energy of the photodiode material $[\mathrm{eV}]$, which is described by the Varshni empirical relation for a Si p-n photodiode case [30], and by the Sajal Paul relation for the $\mathrm{In}_{1-x} \mathrm{Ga}_{x}$ As p-i-n photodiode case [31].

Finally, the total photodiode current at room temperature is given by

$$
\begin{aligned}
I & =I_{P}+I_{D} \\
& =R_{\lambda} \cdot P+C_{n} A_{P} T^{\frac{3}{2}} e^{\left(-\frac{E_{g}(T)}{2 k_{B} T}\right)}\left[e^{\left(\frac{q V_{A}}{k_{B} T}\right)}-1\right] .
\end{aligned}
$$

In the single-pixel camera process, the photocurrent and the dark current have an associated error, due to the discrete nature of the electrical charge [32]. The noise of the former one is known as the photocurrent shot noise $\left(\sigma_{P}\right)$ and it is given by

$$
\sigma_{P}=\sqrt{2 q \bar{I}_{P} B}
$$

where $B$ is the noise bandwidth and $\bar{I}_{P}$ is the photocurrent mean value. The noise of the second current is called the dark current shot noise $\left(\sigma_{D}\right)$, defined as

$$
\sigma_{D}=\sqrt{2 q \bar{I}_{D} B}
$$

where $\bar{I}_{D}$ is the dark current mean value. Note that the photocurrent shot noise depends on the optical signal level and the dark current shot noise does not. The sum of both noise values is known as shot noise $\left(\sigma_{\text {shot }}\right)[26]$ and it follows the Poisson distribution statistics (commonly approximated by a Gaussian distribution when the current is large).

For the sake of completeness, we will consider the JohnsonNyquist (or thermal) noise $\left(\sigma_{\text {thermal }}\right)$, which is produced by the random thermal motion of electrons in a resistor, and it can be modeled as a stationary Gaussian random process (nearly white noise) [26]. The thermal noise is given by [25]

$$
\sigma_{\text {thermal }}=\sqrt{\frac{4 k_{B} T B}{R_{S H}}},
$$

where $R_{S H}$ is the shunt resistance. Since $\sigma_{P}, \sigma_{D}, \sigma_{\text {thermal }}$ are linearly independent noise sources, the total noise $\left(\sigma_{T}\right)$ can be written as

$$
\sigma_{T}=\sqrt{\sigma_{P}^{2}+\sigma_{D}^{2}+\sigma_{\text {thermal }}^{2}}
$$

Consequently, the signal-to-noise ratio (SNR) of the electrical signal in decibels is defined as [26],

$$
S N R=20 \log \left(\frac{\bar{I}_{P}}{\sqrt{2 q \bar{I}_{P} B+2 q \bar{I}_{D} B+\frac{4 k_{B} T B}{R_{S H}}}}\right) .
$$

\section{Numerical model of the single-pixel cam- era}

In this Section, a numerical model of the single-pixel camera is described based on characteristics of the light source and the photodiode. The camera model takes in account the optical power level $\left(P_{i n p}\right)$ and the wavelength $\left(\lambda_{s}\right)$ of the incident light, which is assumed to be monochromatic. It also considers the photocurrent, the dark current, the photocurrent shot noise, the dark current shot noise, and the thermal noise as a function of the photodiode parameters.

In this model, the structured light patterns are 2-D functions $H_{k}(n, n)$ pertaining to the orthonormal WalshHadamard $(\mathrm{WH})$ basis [33]. $H_{k}(n, n)$ are binary matrices whose elements are equal to \pm 1 , where $k=1, \ldots, N^{2}$ denotes the pattern index, $(n, n)$ is the pattern spatial location, and $(N, N)$ are the pattern spatial dimensions. For experimental reasons, in our model $H_{k}(n, n)$ is considered to be composed of a positive $H_{k}^{+}(n, n)$ and a complementary part $H_{k}^{-}(n, n)$, fulfilling the following relation,

$$
H_{k}(n, n)=H_{k}^{+}(n, n)-H_{k}^{-}(n, n),
$$


where $H_{k}^{+}(n, n)$ and $H_{k}^{-}(n, n)$ are complementary matrices whose negative elements are changed to zero. In absence of noise, the mathematical properties of $H_{k}(n, n)$ allow us to recover an exact replica of the object with a 2 -D spatial resolution equal to $(N, N)$ pixels. Fig. (2) shows a schematic representation of one WH pattern.

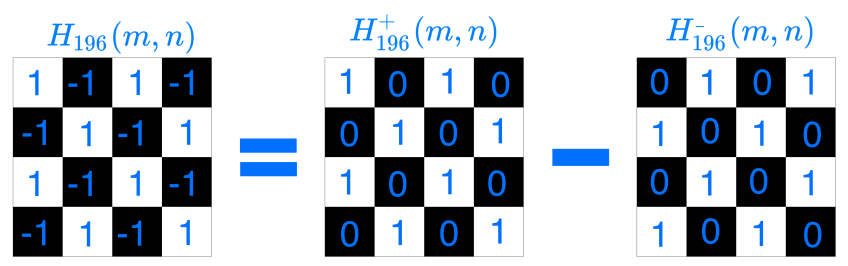

Figure 2: Schematic representation of the positive and the complementary part of a WH pattern with index $k=196$.

The numerical process followed for modeling the singlepixel camera, from the location where the incident light illuminates the spatial light modulator up to where the final image is reconstructed, is as follows:

Step 1 Obtain the number of photons per second $\left(\gamma_{i n p}^{k^{ \pm}}\right)$ corresponding to the wavelength $\left(\lambda_{s}\right)$ and to the optical power of the light source $\left(P_{i n p}\right)$, arriving to the DMD,

$$
\gamma_{i n p}^{k^{ \pm}}=\text {floor }\left(\frac{P_{i n p}}{E_{\gamma}}\right)
$$

where $E_{\gamma}=\frac{h c}{\lambda_{s}}$ is the photon energy.

Step 2 Distribute the $\gamma_{i n p}^{k^{ \pm}}$photons spatially following a statistical Poisson distribution in a matrix $A(n, n)$,

$$
B^{k^{ \pm}}(n, n)=\gamma_{i n p}^{k^{ \pm}} \cdot A(n, n) .
$$

Step 3 Multiply the photon matrix $B^{k^{ \pm}}(n, n)$ by the ideal positive Hadamard pattern $H_{k}^{+}(n, n)$ and its complementary pattern $H_{k}^{-}(n, n)$,

$$
C^{k^{ \pm}}(n, n)=B^{k^{ \pm}}(n, n) \cdot H_{k}^{ \pm}(n, n) .
$$

Step 4 Calculate the number of photons per second $\left(\gamma_{\text {out }}^{k^{ \pm}}\right)$ that strike on the photodiode by projecting $C^{k^{ \pm}}(n, n)$ onto the object $O(n, m)$ as a dot product,

$$
\gamma_{\text {out }}^{k^{ \pm}}=\sum_{m=1}^{M} \sum_{n=1}^{N} C^{k^{ \pm}}(n, n) \cdot O(n, m) .
$$

Step 5 Obtain the optical power $\left(P_{\text {out }}^{k^{ \pm}}\right)$at the photodiode,

$$
P_{\text {out }}^{k^{ \pm}}=\gamma_{\text {out }}^{k^{ \pm}} \cdot E_{\gamma}
$$

Step 6 Calculate the total current as the sum of the photocurrent and the dark current according to Eq.(5),

$$
I^{k^{ \pm}}=R_{\lambda} \cdot P_{\text {out }}^{k^{ \pm}}+C_{n} A_{P} T^{\frac{3}{2}} e^{\left(-\frac{E_{g}(T)}{2 k_{B} T}\right)}\left[e^{\left(\frac{q V_{A}}{k_{B} T}\right)}-1\right] .
$$

Step 7 Obtain the single-pixel noisy current $\left(I_{s p}^{k \pm}\right)$ by adding the photocurrent shot noise, the dark current shot noise and the thermal noise to $I^{k^{ \pm}}$. The noise terms are generated following a Gaussian distribution, taking into account Eqs.(6)-(8), respectively.

Step 8 Obtain the normalized photodetector signal related to $H_{k}(n, n)$ as (taking into account Eq.(11)),

$$
I_{s p}^{k}=\frac{1}{I_{0}}\left(I_{s p}^{k^{+}}-I_{s p}^{k^{-}}\right) .
$$

where $I_{0}$ is the signal measured by the photodiode when the object is illuminated with an uniform pattern.

Step 9 Calculate the single-pixel image $I(n, n)$ multiplying the single-pixel signal by the WH basis as follows

$$
I(n, n)=\frac{1}{N^{2}} \sum_{k=1}^{N^{2}} I_{s p}^{k} \cdot H_{k}(n, n) .
$$

\section{Results}

\subsection{Numerical results}

The numerical model described in the previous section was used to analyze the performance of a single-pixel camera with photodiode detectors under different circumstances. Three different studies were developed analyzing the quality of the final image when: 1) the optical power of the light source changes; 2) we use light sources with different wavelengths; and 3) the photodiode temperature varies.

The simulations were performed for two commercial photodiodes, Thorlabs DET36A and Thorlabs DET10C, whose specifications are shown in Table 1. Moreover, the dark current $\left(I_{D}\right)$, the dark current shot noise $\left(\sigma_{D}\right)$, and the thermal noise $\left(\sigma_{\text {thermal }}\right)$ curves are plotted versus temperature (see Fig. 3). The curves were obtained taking into account the Varshni empirical relation [30] for the Si detector case, and the Sajal Paul relation [31] for the InGaAs detector case. 
Table 1: Photodiode parameters data

\begin{tabular}{l|l|l|l}
\hline Parameter & Symbol & Silicon Biased Detector & $\operatorname{In}_{0.53} \mathrm{Ga}_{0.47} \mathrm{As}_{\text {Biased Detector }}$ \\
\hline Detector Name & & Thorlabs DET36A & Thorlabs DET10C \\
Photodiode active area & $A_{P}$ & $13.0 \mathrm{~mm}^{2}$ & $0.8 \mathrm{~mm}^{2}$ \\
Wavelength range & & $350-1100 \mathrm{~nm}$ & $900-1700 \mathrm{~nm}$ \\
Band gap energy at 298K & $E_{g}$ & $1.1114 \mathrm{eV}$ & $0.7379 \mathrm{eV}$ \\
Rise time response & $t_{r}$ & $14.0 \mathrm{~ns}$ & $10.0 \mathrm{~ns}$ \\
Noise Bandwidth & $B$ & $0.025 \mathrm{nHz}$ & $0.035 \mathrm{nHz}$ \\
Bias Voltage & $V_{A}$ & $10.0 \mathrm{~V}$ & $5.0 \mathrm{~V}$ \\
Saturation current at 298K & $I_{s}$ & $0.35 \mathrm{nA}$ & $1.0 \mathrm{nA}$ \\
Shunt resistance & $R_{s h}$ & $1.0 \mathrm{G} \Omega$ & $10.0 \mathrm{G} \Omega$ \\
NEP at $\lambda_{P}$ & & $1.6 \times 10^{-14} \frac{W}{\sqrt{H z}}$ & $2.5 \times 10^{-14} \frac{W}{\sqrt{H z}}$ \\
\hline
\end{tabular}

(a)

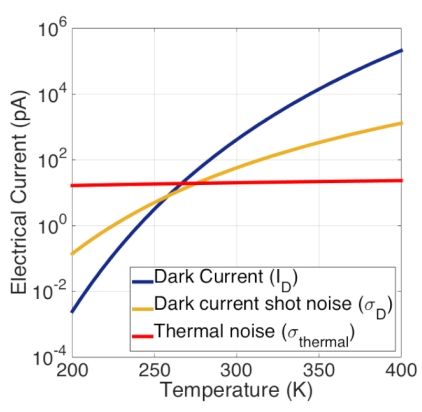

(b)

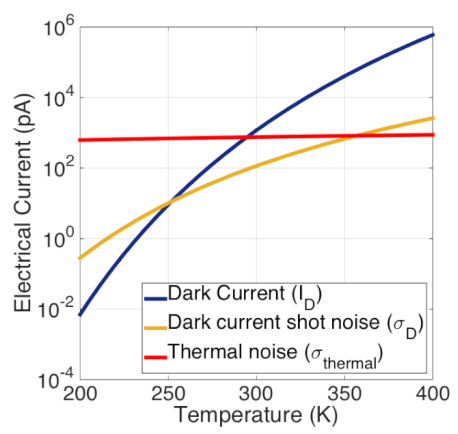

Figure 3: Simulated dark current, dark current shot noise and the thermal noise as a functions of the temperature for (a) $\mathrm{Si}$ biased detector (Thorlabs DET36A) and (b) InGaAs biased detector (Thorlabs DET10C).

In order to evaluate the quality of the images, we used the signal-to-noise ratio (SNR) metric defined by [34],

$$
S N R=10 \log _{10}\left[\frac{\sum_{n=1}^{N^{2}}\left[I_{R}(n, n)\right]^{2}}{\sum_{n=1}^{N^{2}}\left[I_{R}(n, n)-I(n, n)\right]^{2}}\right],
$$

where $I_{R}(n, n)$ is the reference image and $I(n, n)$ is the retrieved image. The reference image is obtained by singlepixel imaging techniques but using only the photocurrent values without considering the noise sources.

In our first study of the single-pixel camera, we analyzed the image quality as a function of the optical power of the light source. We fixed the wavelength of the light source to $520 \mathrm{~nm}$ and the photodiode temperature to $298 \mathrm{~K}$. As a first example, Fig. 4 shows the photocurrent, the dark current, and the total current associated to the single-pixel camera for two different optical power levels; (a) $42.49 \mu \mathrm{W}$ and (b) $0.0085 \mu \mathrm{W}$, respectively. We also show images reconstructed by applying single-pixel imaging techniques to the different current signals in the plot. We can see that for (a)

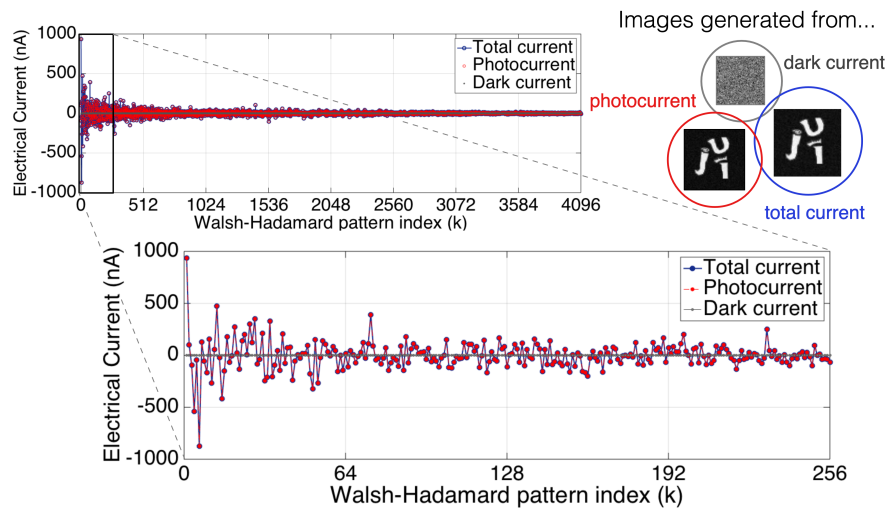

(b)

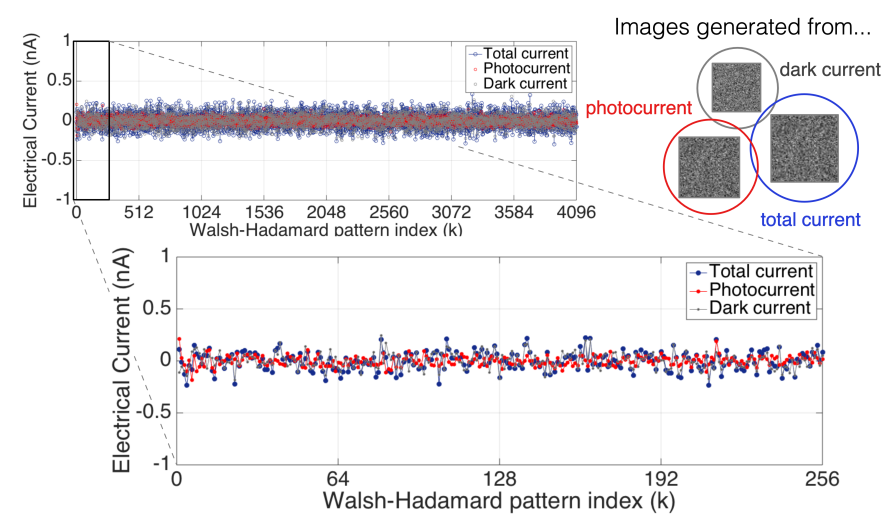

Figure 4: Photocurrent, dark current, and total current with their associated noise values as a function of WH pattern index. Two different optical power values were considered (a) $42.49 \mu \mathrm{W}$ and (b) $0.0085 \mu \mathrm{W}$. The wavelength of the light source was fixed at $520 \mathrm{~nm}$ and the photodiode temperature at $298 \mathrm{~K}$. In each case, the recovered images from these electric signals are shown on the right.

the case of low light power in Fig. 4(b), the photocurrent is noisier, the total current is worst, and therefore the quality of the image deteriorates with respect to the case in Fig. 4(a). To numerically evaluate the quality of the recovered 
image, we plot in Fig. 5 the SNR as a function of the optical power of the light source. As expected, the quality of the image obtained by single-pixel camera improves when the optical power increases. In the same figure, we also plot the curve of the SNR of the photodiode signal as a function of the light power. This SNR curve was calculated by means of Eq.(20) considering the photodiode signal without noise as reference. Of course, the SNR is the same in both cases. Therefore we will use the SNR applied to the images, from now onwards.

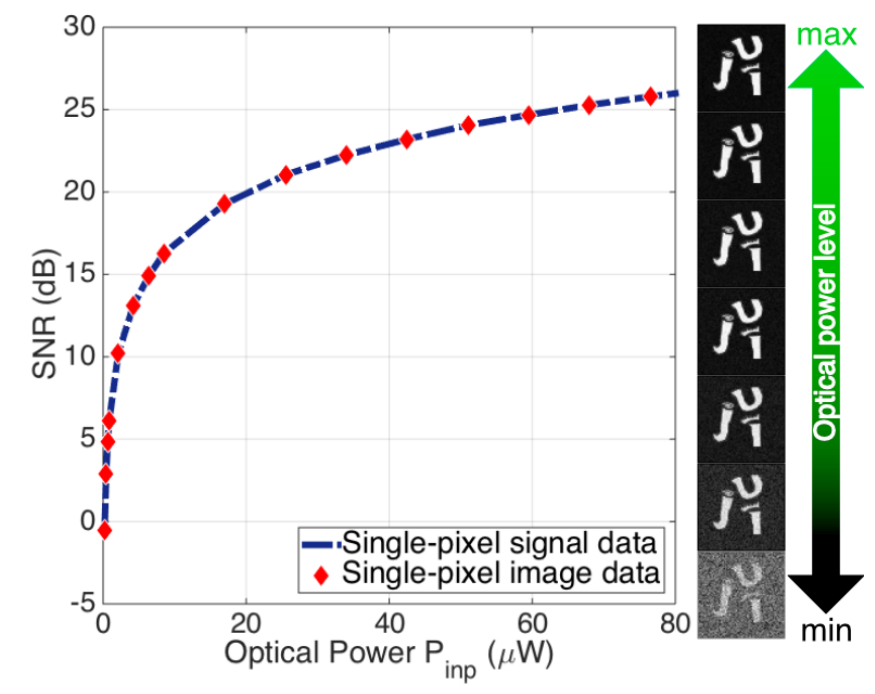

Figure 5: SNR of the signal and the reconstructed images as a function of the optical power $P_{i n p}$. The reconstructed images on the right part are a sample of the SNR red data points.

In the second study we analyzed how the wavelength of the light source influences the performance of the single-pixel camera. The optical power of the light source was set to $8.49 \mu \mathrm{W}$ and the photodiode temperature to $298 \mathrm{~K}$. Fig. 6 (a) shows the dependence of the SNR versus the wavelength for the DET36A and the DET10C photodiodes. In Fig. 6(b) we display several images reconstructed with our model for different wavelengths of the incident light. The key point to understand the relation between the image quality and the wavelength is the photodiode responsivity $\left(R_{\lambda}\right)$. In a photodetector the incident optical power and the generated photocurrent are proportionally related by the responsivity (see Eq.(1)). Therefore the photocurrent increases as the responsivity rises up, although optical power remains constant. The responsivity versus wavelength curves for both photodiodes are presented in Fig. 6(c) $[35,36]$. As we can see comparing Fig. 6(a) and Fig. $6(\mathrm{c})$, the behavior of the SNR and responsivity curves are closely related.

Finally, we analyzed the dependency of the image quality with the photodiode temperature. The wavelength of the light source was set to $520 \mathrm{~nm}$ and $1600 \mathrm{~nm}$ for the DET35A and the DET10C detectors, respectively. For each detector, three curves of the SNR as a function of the photodiode temperature are plotted for fixed values of the optical power $(42.49 \mu W, 8.49 \mu W$ and $0.21 \mu W)$ as shown in Fig. 7. Moreover, several single-pixel images for different values of temperature and optical power are displayed. In general, the SNR of the image decreases as the temperature increases. However, as we can see in the figure, the influence of the temperature on the SNR value is less significant for higher optical power levels. In particular, the performance of these photodiodes is suitable even with high temperatures whenever the optical power is higher than $8.50 \mu \mathrm{W}$. As previously shown in Fig. 3, the dark current and the dark current shot noise increase when the temperature increases. Although the current and its noise increase when the temperature increases, this effect is negligible in the SNR curves if the optical power level is high.

(a)

(b)

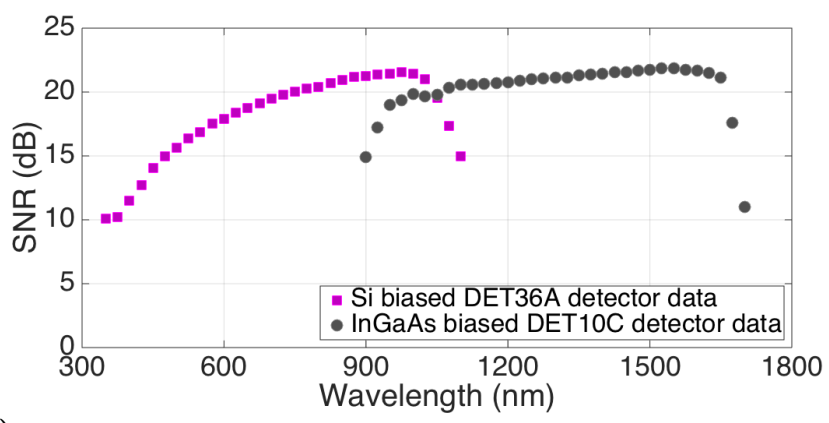

(c)
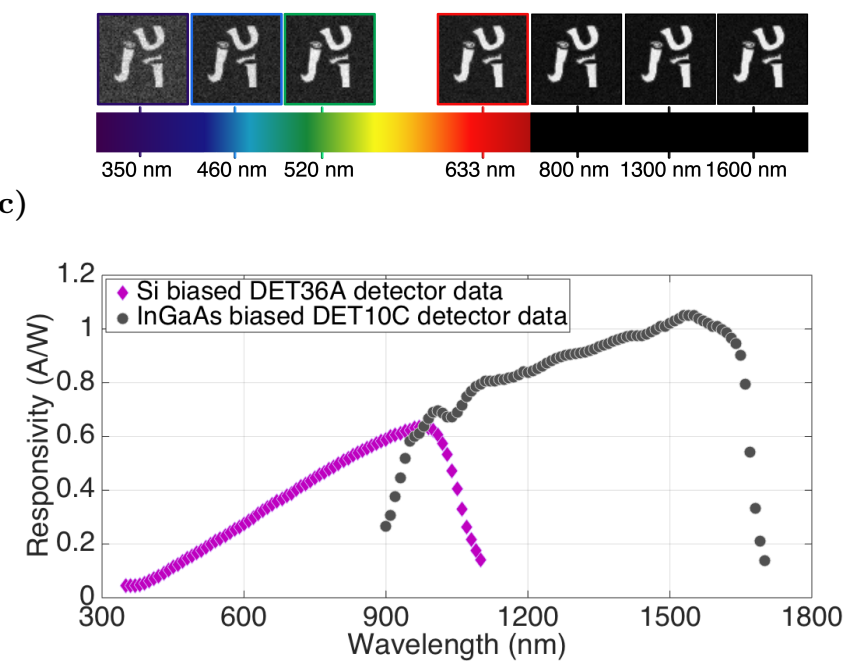

Figure 6: (a) Signal-to-Noise ratio as a function of wavelength of light source, (b) single-pixel images for different wavelengths, and (c) Responsivity data of both photodiodes [35,36]. 
(a)

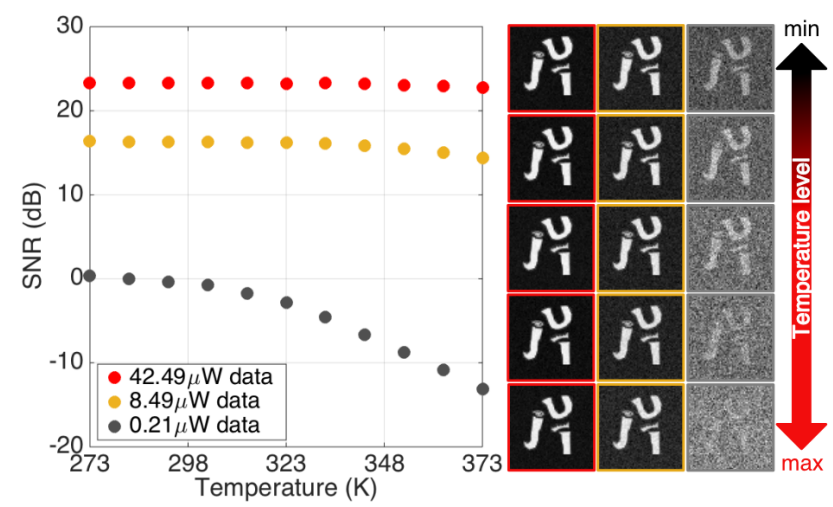

(b)

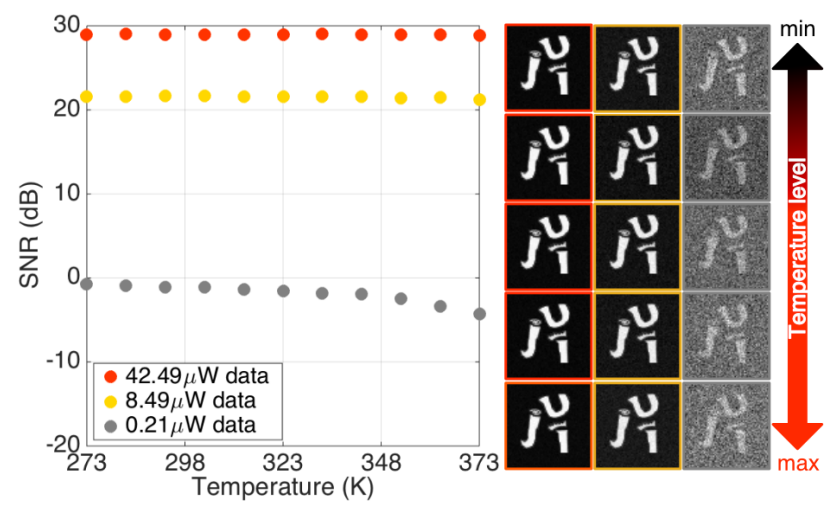

Figure 7: SNR dependence with the photodiode temperature for three optical power levels: $42.49 \mu \mathrm{W}, 8.49 \mu \mathrm{W}$, and $0.21 \mu \mathrm{W}$ for (a) the Si biased detector (Thorlabs DET36A), and (b) the InGaAs biased detector (Thorlabs DET10C). The reconstructed images for those optical power levels are shown as well.

\subsection{Experimental results}

A scheme of the experimental setup by transillumination is depicted in Fig. 1. We used a white-light source with a narrow band pass filter centered at a wavelength of $520 \mathrm{~nm}$ (the same wavelength as that used in Subsection 4.1) and a neutral density filter wheel (NDC-100S-4M-Mounted Step Variable ND Filter) was located in front of the lamp. The object was a black and white logo of our university (UJI) printed in a transparent acetate slide. The object size was $11 \times 11 \mathrm{~mm}$ with a total transmittance factor of 0.12 . It should be mentioned that this object has the same features as the one used in Subsection 4.1. In order to apply singlepixel imaging techniques, we illuminated the object with a sequence of light patterns corresponding to functions of the WH basis, which were generated by a digital micromirror device (DMD). The DMD features were the following: a chipset array size of $1920 \times 1080$ micromirrors with a micromirror pixel pitch of $10.8 \mu \mathrm{m}$ and a display resolution of 1080p (DLP Discovery 4100, Texas Instruments). The WH patterns were displayed in a chip area of 1024 x 1024 micromirrors with a resolution of $64 \times 64$ pixels. Afterwards, we projected them onto the object plane using a 4-f imaging system composed by two achromatic lenses (L1 and L2). The focal distances of L1, and L2 were $f_{1}=100 \mathrm{~mm}$, and $f_{2}=100 \mathrm{~mm}$, respectively. Note that the magnification factor of the 4-f optical system was 1, therefore the field of view (FOV) was $11 \times 11 \mathrm{~mm}$, which is, in fact, the WH patterns size on the DMD display. The light transmitted by the object was subsequently collected by a lens L3 and focused onto a Si biased detector (Thorlabs DET36A). To this end, the detector was located at $f_{3}=50 \mathrm{~mm}$; the focal distance of L3. The incident light power level was changed using the filter wheel and measured with a power meter (Coherent, FieldMaster GS) close to the photodiode sensor. Finally, the signal was digitized and saved in a computer by using a DAQ system. This signal was then used to reconstruct the image, using Eq.(19).

(a)

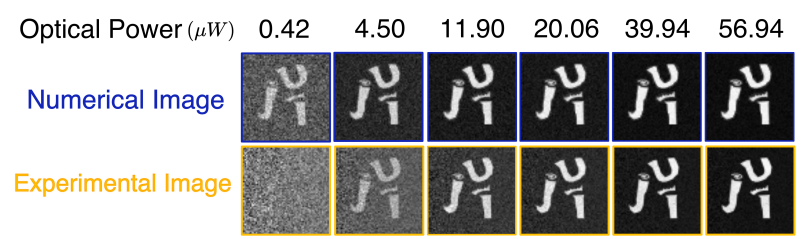

(b)

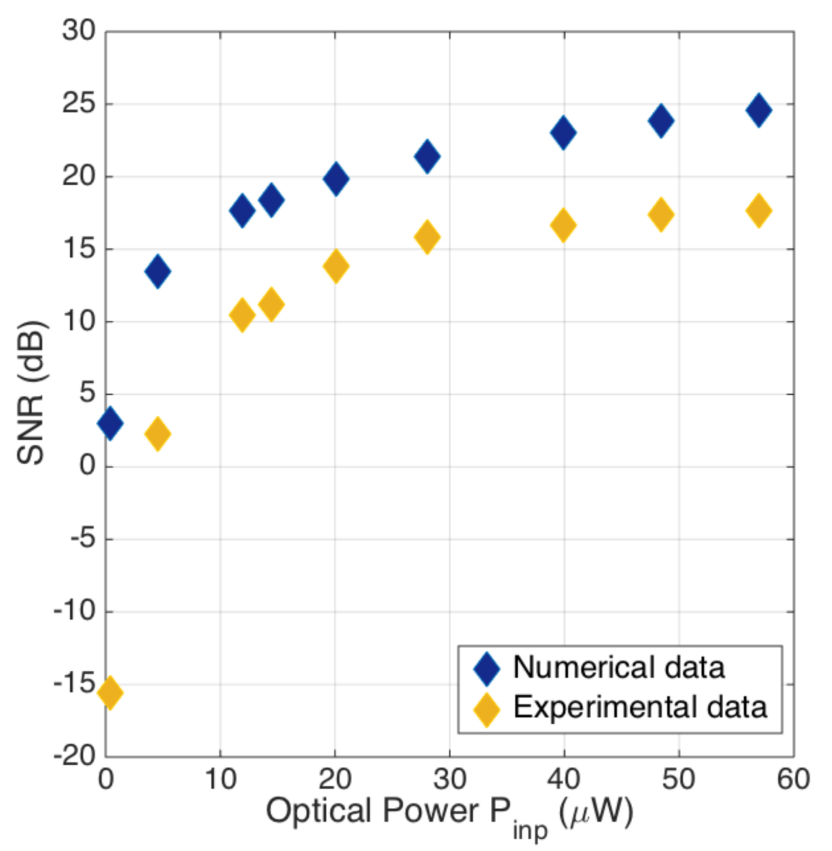

Figure 8: (a) Numerically and experimentally reconstructed images for different optical power levels $P_{i n p}$; (b) SNR dependence with $P_{i n p}$ for the experimental and numerical single-pixel images. 
Fig. 8(a) shows experimentally and numerically reconstructed images for different levels of the optical power. We can see that, in both cases, the noise level decreases with the total optical power. However, the experimental images are noisier than their numerical counterparts. This is corroborated by the results in Fig. 8(b), which shows that the SNR curve corresponding to the numerical and the experimental images have a similar dependence with the optical power. However the experimental SNR function is lower than the numerical one.

Even though the numerical model has been developed taking into account the most important noise factors during the acquisition process, there is a discrepancy in the values of the SNR for the experimental and the simulated images. This error is produced by several other noise sources that can not be easily modeled. For instance, noise introduced by the object substrate, by the signal amplification process, and by the analogue to digital conversion procedure.

\section{Conclusions}

A numerical model of a single-pixel camera has been developed, considering the characteristics of the incident light and the physical properties and specifications of the photodiode. We have accomplished a careful and rigorous mathematical review of the electrical behavior of $\mathrm{Si}$ and InGaAs detectors. Our model takes into account the photocurrent, the dark current, the photocurrent shot noise, the dark current shot noise, and the Johnson-Nyquist (thermal) noise of the Thorlabs DET36A and Thorlabs DET10C detectors, respectively.

Numerical simulations with our model have allowed us to analyze the behavior of the single-pixel imaging technique in different contexts. In particular, we have studied the quality of the final image as a function of the light source power level. We have corroborated the reduction of the SNR for low light levels. We have also observed the clear link between the quality of the photocurrent signal and the quality of the reconstructed image. These results can be useful to predict the behavior of imaging systems working in low light level conditions.

We have also studied the dependence of the SNR with the wavelength. In this case we conclude that the influence of the wavelength arises from the variation of the quantum efficiency and the responsivity of the photodetector. Such analysis could be the first step in the application of singlepixel imaging techniques to multispectral imaging.

Finally, we have analyzed the quality of the images provided by the single-pixel camera as a function of the photodiode temperature. The study is performed for both a
Si biased and an InGaAs biased detectors. The main conclusion in this case is that the SNR of the reconstructed images changes only slightly with the temperature for high values of the light power. However, the reduction is clearly significant for low light levels. Therefore, cooling the detector can play an important role in photon counting or low light level applications.

A single-pixel camera has been developed in order to compare the results provided by our model with those obtained experimentally. We have shown that the quality of the final image, in terms of the SNR, changes in a similar way with the light power. However, the results given by the model and the experiment do not overlap perfectly. The discrepancy might be due to unaccounted uncertainty sources such as noise introduced by the object substrate, the signal amplification process, and the analogue to digital conversion procedure.

\section{Funding Information}

We acknowledge financial support from MINECO (FIS2016-75618-R and FIS2015-72872-EXP), Generalitat Valenciana (PROMETEO/2016/079), and Universitat Jaume I (P1-1B2015-35). Yessenia Jauregui-Sánchez acknowledges the Santiago Grisolía support from Generalitat Valenciana (GRISOLIA/2015/037).

\section{References}

[1] M. F. Duarte, M. A. Davenport, D. Takbar, J. N. Laska, T. Sun, K. F. Kelly, and R. G. Baraniuk, "Single-Pixel Imaging via Compressive Sampling," IEEE Signal Process. Mag. 25, 83-91 (2008).

[2] V. Studer, J. Bobin, M. Chahid, H. S. Mousavi, E. Candes, and M. Dahan, "Compressive fluorescence microscopy for biological and hyperspectral imaging," Proc. Natl. Acad. Sci. USA 109, E1679-E1687 (2012).

[3] F. Soldevila, E. Irles, V. Durán, P. Clemente, M. Fernández-Alonso, E. Tajahuerce, and J. Lancis, "Single-pixel polarimetric imaging spectrometer by compressive sensing," Appl. Phys. B 113, 551-559 (2013).

[4] V. Durán, P. Clemente, M. Fernández-Alonso, E. Tajahuerce, and J. Lancis, "Single-pixel polarimetric imaging," Opt. Lett. 37, 824-826 (2012).

[5] E. J. Candès and M. B. Walkin, "An introduction to compressive sampling," IEEE Signal Process. Mag. 25, 21-30 (2008). 
[6] H. Chen, N. Xi, B. Song, L. Chen, J. Zhao, K. W. C. Lai, and R. Yang, "Infrared camera using a single nano-photodetector," IEEE Sens. J. 13, 949-958 (2013).

[7] G. M. Gibson, B. Sun, M. P. Edgar, D. B. Phillips, N. Hempler, G. T. Maker, G. P. A. Malcolm, and M. J. Padgett, "Real-time imaging of methane gas leaks using a single-pixel camera," Opt. Express 25, 2998-3005 (2017).

[8] R. I. Stantchev, B. Sun, S. M. Hornett, P. A. Hobson, G. M. Gibson, M. J. Padgett, and E. Hendry, "Noninvasive, near-field terahertz imaging of hidden objects using a single-pixel detector," Sci. Adv. 2, e1600190 (2016).

[9] B. Sun, M. P. Edgar, R. Bowman, L. E. Vittert, S. Welsh, A. Bowman, and M. J. Padgett, "3D computational imaging with single-pixel detectors," Science 340, 844-847 (2013).

[10] J. Hunt, T. Driscoll, A. Mrozack, G. Lipworth, M. Reynolds, D. Brady, and D. R. Smith, "Metamaterial apertures for computational imaging," Science 339, 310-313 (2013).

[11] E. Tajahuerce, V. Durán, P. Clemente, E. Irles, F. Soldevila, P. Andrés, and J. Lancis, "Image transmission through dynamic scattering media by singlepixel photodetection," Opt. Express 22, 1694516955 (2014).

[12] V. Durán, F. Soldevila, E. Irles, P. Clemente, E. Tajahuerce, P. Andrés, and J. Lancis, "Compressive imaging in scattering media," Opt. Express 23, 14424-14433 (2015).

[13] J. Shin, B.T. Bosworth, and M. A. Foster, "Single-pixel imaging using compressed sensing and wavelength-dependent scattering," Opt. Lett. 41, 886-889 (2016).

[14] G. A. Howland, D. J. Lum, M. R. Ware, and J. C. Howell, "Photon counting compressive depth mapping," Opt. Express 21, 23822-23837 (2013).

[15] A. Kirmani, D. Venkatraman, D. Shin, A. Colaço, F. N. C. Wong, J. H. Shapiro, V. K. Goyal, "FirstPhoton Imaging," Science, 343, 58-61 (2014).

[16] M. J. Sun, M. P. Edgar, G. M. Gibson, B. Sun, N. Radwell, R. Lamb, and M. J. Padgett, "Single-pixel three-dimensional imaging with time-based depth resolution," Nat. Commun. 7, 12010 (2016).
[17] E. Salvador-Balaguer, P. Clemente, E. Tajahuerce, F. Pla, and J. Lancis, "Full-color stereoscopic imaging with a single-pixel photodetector," J. Display Technol. 12, 417-422 (2016).

[18] N. Radwell, K. J. Mitchell, G. M. Gibson, M. P. Edgar, R. Bowman, and M. J. Padgett, "Single-pixel infrared and visible microscope," Optica 1, 285-289 (2014.)

[19] A. D. Rodríguez, P. Clemente, E. Tajahuerce, and J. Lancis, "Dual-mode optical microscope based on single-pixel imaging," Opt. Lasers Eng. 82, 87-94 (2016).

[20] N. Huynh, E. Zhang, M. Betcke, S. Arridge, P. Beard, and B. Cox, "Single-pixel optical camera for video rate ultrasonic imaging," Optica 3, 26-29 (2016).

[21] B. Lochocki, A. Gambín, S. Manzanera, E. Irles, E. Tajahuerce, J. Lancis, and P. Artal, "Single pixel camera ophthalmoscope," Optica 3, 1056-1059 (2016).

[22] M. J. Sun, M. P. Edgar, D. B. Phillips, G. M. Gibson, and M. J. Padgett, "Improving the signal-tonoise ratio of single-pixel imaging using digital microscanning," Opt. Express 24, 10476-10485 (2016)

[23] C. B. Xue, X. R. Yao, X. F. Liu, G. J. Zhai, Q. Zhao, and X. Y. Guo, "Improving the signal-to-noise ratio of complementary compressive imaging with a threshold," Opt. Commun. 393, 118-122 (2017).

[24] M. J. Sun, Z. H. Xua, and L. A. Wu, "Collective noise model for focal plane modulated single-pixel imaging," Opt. Lasers Eng. 100, 18-22 (2018).

[25] S. M. Sze and K. K. Ng, Physics of Semiconductor Devices (John Wiley \& Sons Inc., 2007).

[26] G. P. Agrawal, Fiber-optic communication systems (John Wiley \& Sons Inc., 2010).

[27] T. Pearsall, M. Piskorski, A. Brochet, and J. Chevrier, "A Ga0.47In0.53As/InP heterophotodiode with reduced dark current," IEEE J. Quantum Electron. 17, 255-259 (1981).

[28] K. Ohnaka, M. Kubo, J. Shibata, "A Low Dark Current InGaAs/InP p-i-n Photodiode with Covered Mesa Structure," IEEE Trans. Electron Devices 34, 199-204 (1987).

[29] B. Jacob, B. Witzigmann, M. Klemenc, and C. Petit, "A TCAD methodology for high-speed photodetectors," Solid-State Electron. 49, 1002-1008 (2005). 
[30] I. Vurgaftman, J.R. Meyer and L.R. Ram-Mohan, "Band parameters for III-V compound semiconductors and their alloys," J. Appl. Phys. 89, 5815-5875 (2001).

[31] Sajal Paul, J. B. Roy, and P. K. Basu, "Empirical expressions for the alloy composition and temperature dependence of the band gap and intrinsic carrier density in $G a_{x} I n_{1-x} A s$," J. Appl. Phys. 69, 827-830 (1991).

[32] W. R. Bennett, "Sources and properties of electrical noise," Electrical Engineering, 73, 1001-1006 (1954).

[33] W. Pratt, J. Kane, and H. Andrews, "Hadamard transform image coding," Proc. IEEE 57, pp. 58-68 (1969).

[34] R. C. Gonzalez and R. E. Woods, Digital Image Processing (Addison-Wesley, 2008).

[35] Thorlabs, Inc., "Si Biased Detector Responsivity (DET36A)," http://www.thorlabs.com/ newgrouppage9.cfm?objectgroup_id=1295.

[36] Thorlabs, Inc., "InGaAs Biased Detector Responsivity (DET10C)," http://www.thorlabs.com/ newgrouppage 9. cfm?objectgroup_id=1295. 